\title{
Loss of SDC1 Expression Is Associated with Poor Prognosis of Colorectal Cancer Patients in Northern China
}

\author{
Kaizhi Li, Lei Li, Xiaoxiao Wu, Juan Yu, Hongjun Ma, Renya Zhang $\mathbb{D}^{\text {, }}$, Yan Li $\mathbb{D}$, \\ and Wei Wang iD \\ Department of Pathology, Affiliated Hospital of Jining Medical University, Jining Medical University, Jining, \\ Shandong 272029, China \\ Correspondence should be addressed to Renya Zhang; Zhangrenya@mail.Jnmc.edu.cn, Yan Li; doctor_ly@163.com, \\ and Wei Wang; weiwangphd@163.com
}

Received 3 August 2018; Revised 5 January 2019; Accepted 31 January 2019; Published 30 April 2019

Academic Editor: Matteo Giulietti

Copyright $\odot 2019$ Kaizhi Li et al. This is an open access article distributed under the Creative Commons Attribution License, which permits unrestricted use, distribution, and reproduction in any medium, provided the original work is properly cited.

Background. Syndecan-1 (SDC1/CD138) is a key cell surface adhesion molecule essential for maintaining cell morphology and the interactions with the surrounding microenvironment. SDC1 tumor immunoexpression may be increased or decreased in epithelial malignant neoplasms compared to that in adjacent non-neoplastic tissue, depending on the type of carcinoma, and it has been correlated with various clinicopathological parameters and patient prognosis. SDC1 expression is decreased in colorectal cancer (CRC) tissue, but the relationship between prognosis and SDC1 expression in CRC patients is controversial. Methods. In this study, SDC1 expression was detected in 65 adjacent non-neoplastic colorectal tissues, 477 CRCs, and 79 metastatic lymph nodes using tissue microarray. Results. The data show that SDC1 decreased in CRC tissues $(p \leq 0.001)$ and metastatic lymph node tissues $(p \leq 0.001)$ compared to that in adjacent non-neoplastic colorectal tissues. Loss of SDC1 protein expression is associated with poor overall $(p<0.0001)$ and disease-free survival $(p<0.0001)$, differentiation $(p=0.017)$, stage $(p \leq 0.001)$, and lymph node metastasis $(p \leq 0.001)$ in CRC patients. Conclusions. These data suggest that the loss of SDC1 plays an important role in CRC malignant progression. Loss of SDC1 expression indicates poor prognosis in patients from northern China with CRC.

\section{Introduction}

Colorectal cancer (CRC) is the most common tumor of the gastrointestinal system and ranks as the fourth leading cause of cancer-related deaths [1]. The highest incidence rates of $\mathrm{CRC}$ are observed in Europe, North America, and Oceania; the lowest rates are reported in Asia, Africa, and South America [2].

SDC1 (syndecan-1, CD138), an important cell adhesion molecule, belongs to the family of syndecans, which are transmembrane heparan sulfate proteoglycans (HSPG) [3]. SDC1 is expressed predominantly in epithelial cells, but it is also found in fibroblasts, myoblasts, and differentiating B cells [4-6]. SDC1 can be cleaved and thus releases the extracellular (ectodomain) core protein-shed SDC1 [7]. The shed SDC1 is increased in response to growth factors, chemokines, heparanase, microbial toxins, insulin, and cellular stress $[8,9]$. Although the high shed SDC1 levels in serum have been associated with poor prognosis of CRC patients [10], the relationship between prognosis and epithelial SDC1 expression levels in CRC is controversial [4-6].

In this study, SDC1 expression was detected in 65 adjacent non-neoplastic colorectal tissues, $477 \mathrm{CRC}$ tissues, and 79 metastatic lymph node tissues. The aim of this study was to evaluate the relationship between SDC1 expression and the prognosis of CRC patients from China.

\section{Materials and Methods}

2.1. Colorectal Biopsy Specimens. A cohort of 477 (477/621, $76.8 \%)$ subjects with CRC, 65 (65/621, 10.5\%) adjacent non-neoplastic colorectal epithelia control subjects, and 79 $(79 / 621,12.7 \%)$ subjects with metastatic lymph nodes were recruited between 2008 and 2014 from the Department of 
Gastrointestinal Surgery in the Affiliated Hospital of Jining Medical University (Shandong, PR China). Of the 477 CRC patients, 250 (52.4\%) were male and 227 (47.6\%) were female (with a mean age of 61 years). All biopsies were immediately fixed in $4 \%$ buffered paraformaldehyde and were routinely processed. Tumors were classified according to the standard TNM staging guidelines of UICC (TNM Classification of Malignant Tumours Eighth Edition). All patients had longterm follow-up results. A cohort of 8 fresh CRC biopsies and paired, adjacent non-neoplastic colorectal tissue samples were collected from patients from the Affiliated Hospital of Jining Medical University. The study protocol was reviewed and approved by the local ethics committee. All patients gave written consent for the tissue samples.

2.2. TMA Construction. Representative areas of the CRC, adjacent non-neoplastic colorectal epithelia, and metastatic lymph node tissues were marked on each hematoxylineosin (H\&E) slide. The TMAs were assembled with a tissuearraying instrument (Beecher Instruments, Silver Springs, MD, USA) as described by Kallioniemi et al. [11].

2.3. Immunohistochemical Staining. Immunohistochemical staining of the SDC1 protein was performed on the TMA slides using the streptavidin-peroxidase (S-P) method as previously described [12]. Briefly, each TMA section was deparaffinized and rehydrated. Antigen retrieval was performed at $95^{\circ} \mathrm{C}$ in $1 \mathrm{x}$ EDTA (ethylenediaminetetraacetic acid) buffer (pH 9.0) for $15 \mathrm{~min}$. Inactivation of endogenous peroxidase was performed by using $0.3 \% \mathrm{H}_{2} \mathrm{O}_{2}$-methanol for $30 \mathrm{~min}$. Nonspecific binding was prevented by incubation with normal serum for $20 \mathrm{~min}$ at room temperature (RT), followed by incubation with the primary monoclonal antibody against human SDC1 (dilution 1:100, Clone No. MI15, Fuzhou Maixin Biotech. Co. Ltd., China) at $4^{\circ} \mathrm{C}$ overnight. Antibody binding was detected using EnVision reagents (Dako REAL EnVision Detection System; peroxidase/DAB1, DakoCytomation, Denmark). The immune reaction was visualized by incubation with 3,30-diaminobenzidine chromogen substrate (DAB1 Chromogen, DAKOVR, Carpinteria, CA, USA) for 10 min at RT. Finally, slides were counterstained with hematoxylin-eosin, dehydrated, and coverslipped with a mounting automat (Sakura GLC 550, Tissue-TekVR, Alphen aan den Rijn, The Netherlands). SDC1 expression was scored by two independent pathologists without prior knowledge of patients' clinicopathological characteristics. Three nonmetastatic lymph nodes were used as negative ( $\mathrm{T}$ and $\mathrm{B}$ cells) and positive (plasmocytes) controls for SDC1 staining. The color photomicrographs were taken with an upright metallurgical microscope. In tumors (adjacent non-neoplastic glandular epithelium and metastatic lymph nodes), immunohistochemical reactions were classified for intensity as previously described $[13,14]$. Briefly, low expression ( $L$, - or \pm ), no staining (-), weak staining $( \pm)$, or strong staining was observed in less than $25 \%$ of tumor cells. Moderate expression $(\mathrm{M},+)$, moderate staining, or strong staining was observed in only $25-75 \%$ of tumor cells. High expression $(\mathrm{H},++)$ and strong staining were observed in more than $75 \%$ of tumor cells.
2.4. Protein Extraction and Western Blot. Fresh CRC samples and paired, adjacent non-neoplastic colorectal tissues were homogenized in RIPA lysis buffer (Solarbio, Beijing, China) containing phenylmethylsulfonyl fluoride (PMSF) (SigmaAldrich Corporation, St Louis, MO, USA). Equal amounts of protein samples were separated by sodium dodecyl sulfate polyacrylamide gel electrophoresis (SDS-PAGE) and were transferred onto a PVDF membrane (Millipore, Billerica, MA, USA). The membranes were immunoblotted with the following antibodies: monoclonal anti-SDC1 (dilution $1: 1000$, Clone No. 4H5H5) and anti-GAPDH antibodies (dilution 1:3000, Proteintech Group Inc., Chicago, IL, USA). The immunoreaction was visualized with enhanced chemiluminescence solution (Millipore, Billerica, MA, USA).

2.5. RNA Isolation and Quantitative Real-Time PCR ( $q R T$ $P C R)$. Total RNA was isolated from fresh CRC samples and paired, adjacent non-neoplastic colorectal tissues using TRIzol Reagent (Invitrogen, San Diego, CA, USA) and then treated with DNase (Roche Diagnostics, Rotkreuz, Switzerland) to eliminate contaminating DNA. Next, $1 \mu \mathrm{g}$ of the total RNA sample was reverse-transcribed into cDNA using M-MLV reverse transcriptase according to the manufacturer's instructions (Promega, Madison, WI, USA). qRT-PCR was performed using a Bio-Rad iQ SYBR Green Supermix kit and the Bio-Rad iCycler iQ system (Bio-Rad, Hercules, CA, USA). Human SDC1 primers were used with the forward sequence $\left(5^{\prime}-3^{\prime}\right)$ TGGGGATGACTCTGACAACT and the reverse sequence $\left(5^{\prime}-3^{\prime}\right)$ CACTTCTGGCAGGACT ACAG. Human GAPDH primers were used with the forward sequence $\left(5^{\prime}-3^{\prime}\right)$ AACGGATTTGGTCGTATTGG and the reverse sequence $\left(5^{\prime}-3^{\prime}\right)$ TTGATTTTGGAGGGATCTCG. The expression levels of amplified genes were normalized to GAPDH and were presented as relative expression levels.

2.6. Statistical Analysis. Pearson's $\chi^{2}$ test was used to analyze the association between SDC1 expression and clinicopathological characteristics by using the SPSS 13.0 software package (SPSS, Chicago, IL). The Kaplan-Meier method was used to determine the probability of survival, and GraphPad Prism software (version 6, La Jolla, CA, USA) was used to analyze the data with the log-rank test. Differences in quantitative variables between groups were analyzed by Student's $t$-test and Mann-Whitney test (nonparametric text, data do not assume Gaussian distributions). In the analyses, a $p$ value of $<0.05$ was considered significant.

\section{Results}

3.1. SDC1 Expression Decreased in CRCs and Metastatic Lymph Nodes. We measured SDC1 protein levels by Western blot and mRNA levels by qRT-PCR in 8 fresh CRC samples and paired, adjacent non-neoplastic colorectal tissues. As shown in Figure 1, three expression forms of SDC1 protein were detected, SDC1 protein dimer expression was high (the main expression form), tetramer expression was weak, and monomer expression was absent in all the samples (Figure 1(a)). SDC1 protein was highly expressed in adjacent 


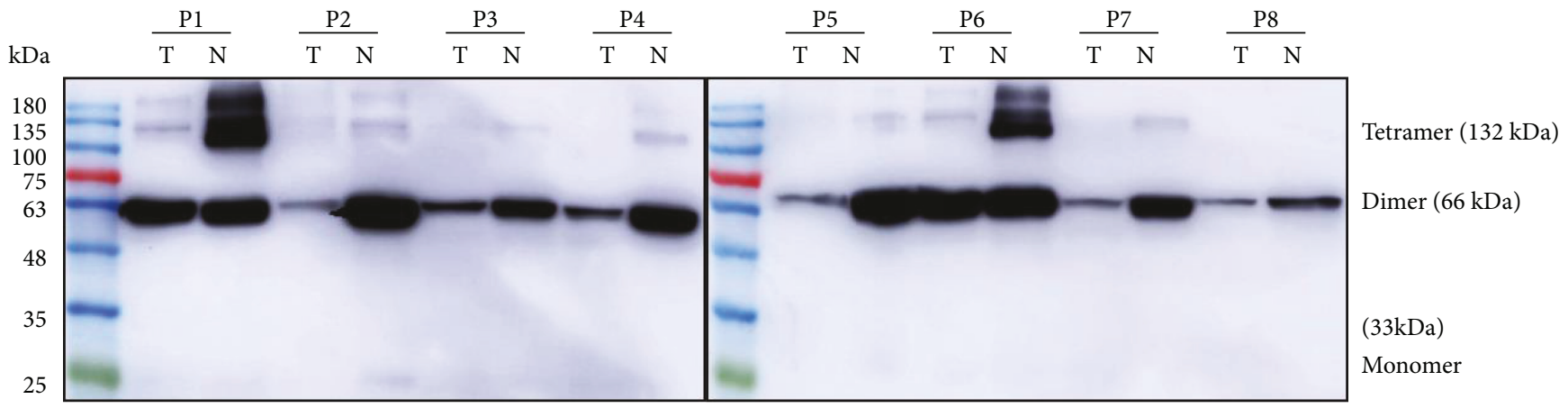

35

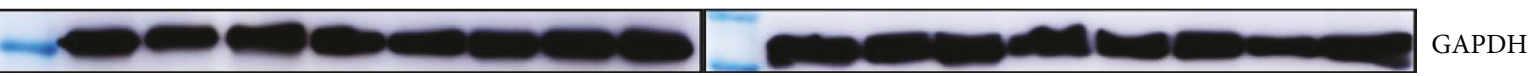

(a)

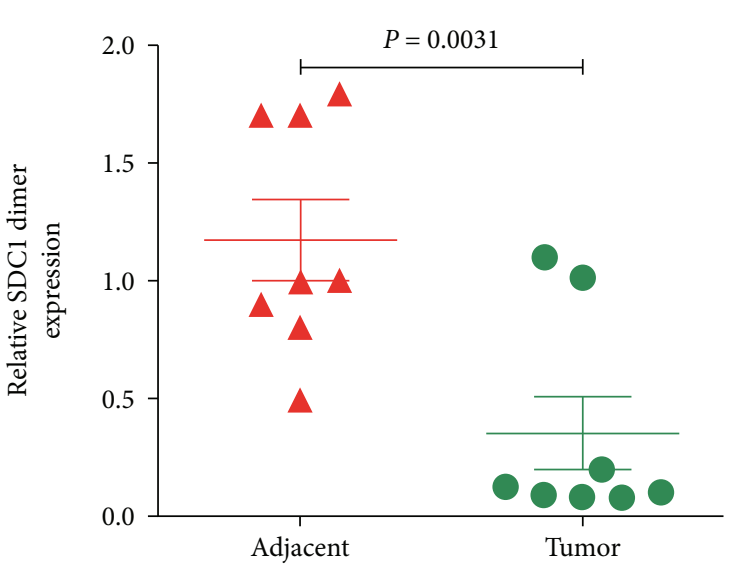

(b)

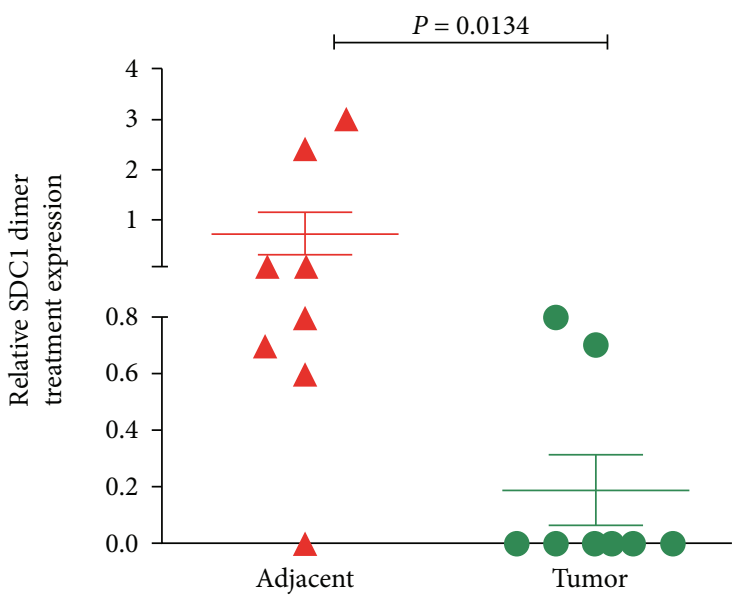

(c)

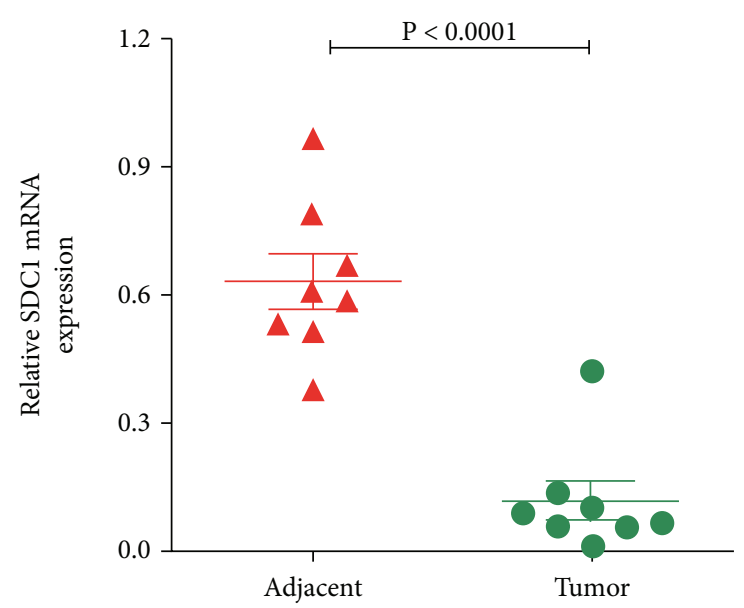

(d)

FIGURE 1: SDC1 expression is decreased in CRCs. (a) Protein expression levels were evaluated in colorectal biopsies taken from 8 tumors (CRCs, T) and adjacent non-neoplastic controls (N) by Western blot. GAPDH was used as a loading control. Quantitative analysis of SDC1 protein: (b) dimer level ( $p=0.0031$, Student's $t$-test) and (c) tetramer level $(p<0.0001$, Mann-Whitney test) in CRC and paired, adjacent non-neoplastic controls. (d) mRNA expression levels were evaluated in colorectal biopsies and adjacent non-neoplastic controls by qRT-PCR. GAPDH was used as a control. $p<0.0001$, Student's $t$-test.

non-neoplastic colorectal tissue homogenates but was weak or undetectable in CRC tissues (Figure 1(b), dimer, $p=$ 0.0031 ; Figure 1(c), tetramer, $p=0.0134)$; SDC1 mRNA expression was decreased in CRC tissues compared to that in paired, adjacent non-neoplastic colorectal tissues (Figure 1(d), $p<0.001)$.

Furthermore, we detected SDC1 expression in 621 cases: 477 samples of CRCs (Figure 2(a), high expression; 


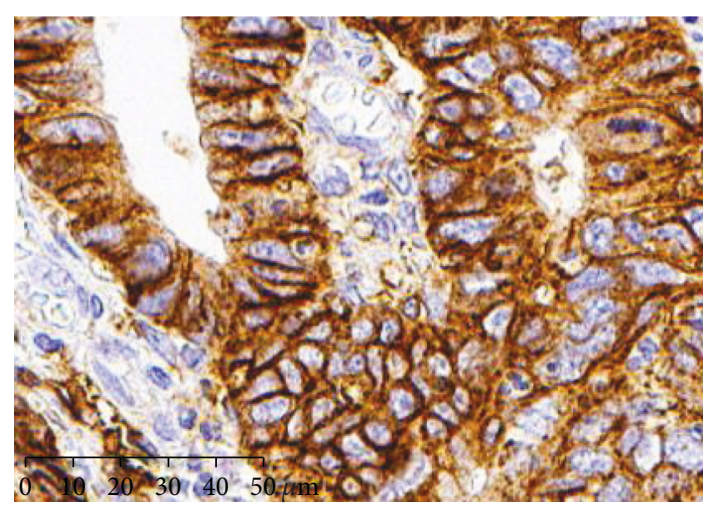

(a)

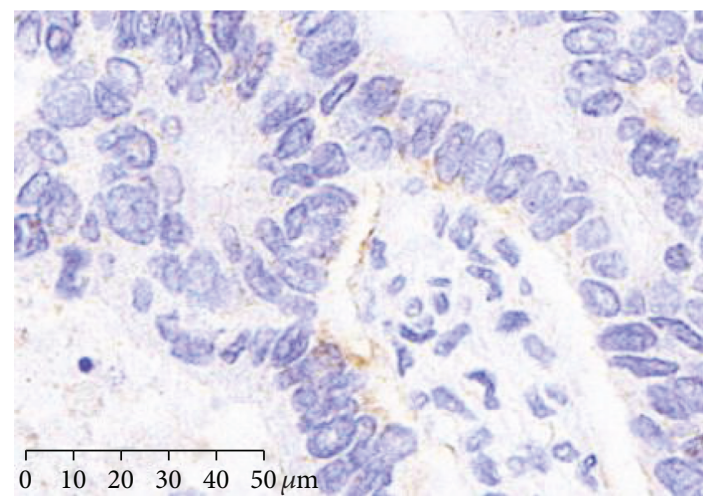

(c)

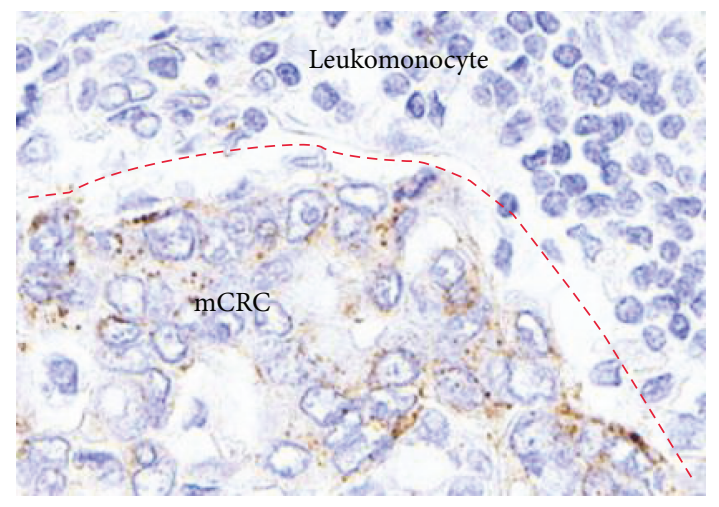

(e)

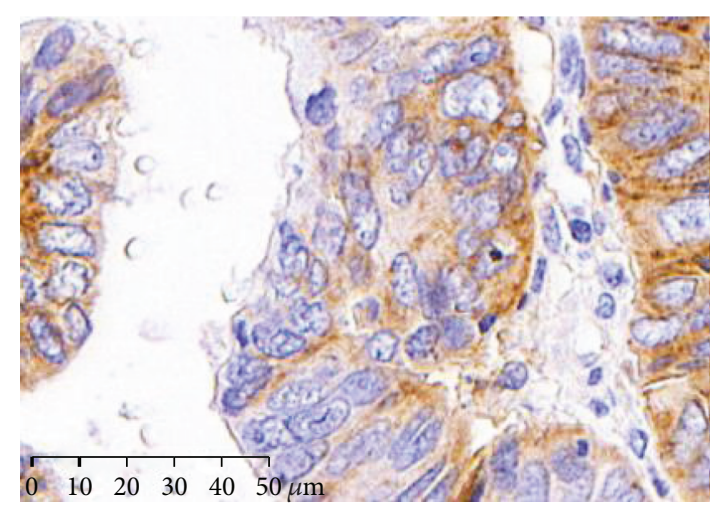

(b)

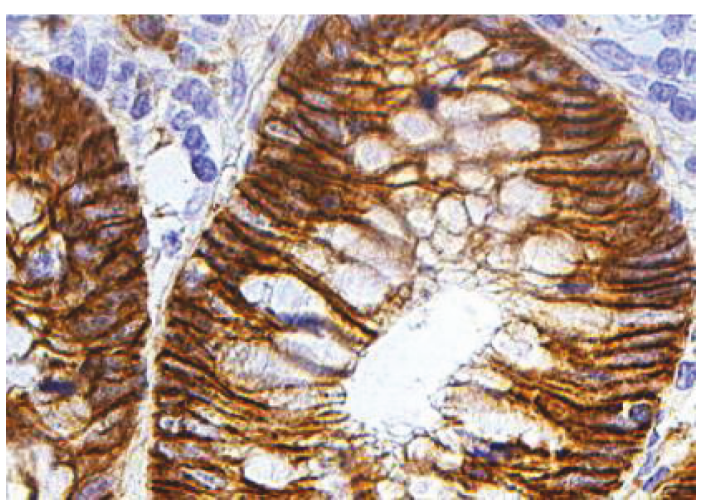

(d)

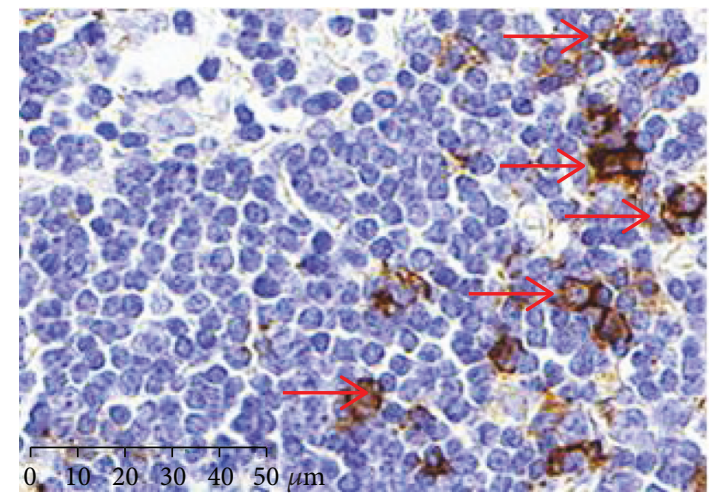

(f)

FIgURE 2: SDC1 decreased both in CRCs and metastatic lymph nodes (mCRC). Immunohistochemical staining for SDC1 in CRCs. (a) High expression, (b) moderate expression, (c) low expression, (d) adjacent non-neoplastic colonic epithelium (high expression), and (e) metastatic lymph nodes (low expression). (f) SDC1 staining in nonmetastatic lymph node as negative control (nonstaining cells, T and B cells) and positive control (red arrow, plasmocytes). Scale bars: $50 \mu \mathrm{m}$.

2(b), moderate expression; and 2(c), low expression), 65 samples of adjacent non-neoplastic colorectal epithelium (Figure 2(d)), and 79 samples of metastatic lymph nodes (Figure 2(e)) from CRCs by immunohistochemistry (IHC). IHC revealed that high SDC1 expression in adjacent nonneoplastic colorectal epithelial cells was detected mainly in the membrane and cytoplasm (Figure 2(d)). In contrast, SDC1 expression was reduced or undetectable in CRC tissues and metastatic lymph nodes from CRCs. Statistically, among the 65 adjacent non-neoplastic colorectal epithelium samples, 57 (88\%) samples showed high expression of SDC1, and $8(12 \%)$ samples showed moderate expression of SDC1.
Only 109 of $477(22.8 \%)$ tissues and 228 of $477(44.8 \%)$ CRC samples exhibited high or moderate SDC1 expression, respectively. The immunointensity of SDC1 in metastatic lymph nodes from CRCs further decreased to lower levels since only 11 of 79 (14\%) samples showed high SDC1 expression (Table 1). Thus, these data indicate that the loss of SDC1 expression is involved in the development and progression of CRC in northern China.

3.2. Association between SDC1 Expression in CRC Tissues and Patients' Clinicopathological Characteristics. According to the SDC1 staining intensity and the extent of positive tumor 
TABLE 1: Loss of SDC1 expression in CRCs and metastatic lymph nodes, 621 cases.

\begin{tabular}{|c|c|c|c|c|c|c|}
\hline & $\begin{array}{c}\mathrm{SDC}^{\mathrm{L}} \\
N=180 \\
\text { Case }(\%)\end{array}$ & $\begin{array}{l}\mathrm{SDC1}^{\mathrm{M}} \\
N=264 \\
\text { Case (\%) }\end{array}$ & $\begin{array}{c}\mathrm{SDC}^{\mathrm{H}} \\
N=177 \\
\text { Case }(\%)\end{array}$ & Total & $\chi^{2}$ & $p$ \\
\hline Adjacent non-neoplastic tissues ${ }^{a}$ & $0(0)$ & $8(12)$ & $57(88)$ & 65 & 114.154 & $\leq 0.001^{\mathrm{ab}}$ \\
\hline $\mathrm{CRCs}^{\mathrm{b}}$ & $140(29.4)$ & $228(47.8)$ & $109(22.8)$ & 477 & 151.685 & $\leq 0.001^{\mathrm{bc}}$ \\
\hline Metastatic lymph nodes ${ }^{c}$ & $40(51)$ & $28(35)$ & $11(14)$ & 79 & 81.639 & $\leq 0.001^{\mathrm{ac}}$ \\
\hline
\end{tabular}

CRC: colorectal cancer; L: low expression; M: moderate expression; H: high expression; statistical method: chi-square test.

TABLE 2: Relationship between SDC1 immunoreactivity and clinicopathological characteristics, 477 CRC cases.

\begin{tabular}{|c|c|c|c|c|c|c|}
\hline Clinical information & Total & $\begin{array}{c}\mathrm{SDC}^{\mathrm{L}} \\
N=140 \\
\text { Case }(\%)\end{array}$ & $\begin{array}{l}\mathrm{SDC1}^{\mathrm{M}} \\
N=228 \\
\text { Case }(\%)\end{array}$ & $\begin{array}{c}\mathrm{SDC}^{\mathrm{H}} \\
N=109 \\
\text { Case (\%) }\end{array}$ & $\chi^{2}$ & $p$ \\
\hline \multicolumn{7}{|l|}{ Age, $y r$} \\
\hline$<60.5$ & 211 & $67(32)$ & $93(44)$ & $51(24)$ & \multirow{2}{*}{2.13} & \multirow{2}{*}{0.345} \\
\hline$>60.5$ & 266 & $73(27)$ & $135(51)$ & $58(22)$ & & \\
\hline \multicolumn{7}{|l|}{ Gender } \\
\hline Female & 227 & $69(30)$ & $110(49)$ & $48(21)$ & \multirow{2}{*}{0.752} & \multirow{2}{*}{0.686} \\
\hline Male & 250 & $71(28)$ & $118(47)$ & $61(24)$ & & \\
\hline \multicolumn{7}{|l|}{ Tumor size, $\mathrm{cm}$} \\
\hline$\leq 4$ & 306 & $92(30)$ & $138(45)$ & $76(25)$ & \multirow{2}{*}{2.924} & \multirow{2}{*}{0.232} \\
\hline$>4$ & 171 & $48(28)$ & $90(53)$ & $33(19)$ & & \\
\hline \multicolumn{7}{|c|}{ Histological grade (differentiation) (miss samples, $N=16$ ) } \\
\hline Well & 226 & $75(33)$ & $111(49)$ & $40(18)$ & \multirow{2}{*}{8.12} & \multirow{2}{*}{0.017} \\
\hline Moderately or poorly & 235 & $59(25)$ & $110(47)$ & $66(28)$ & & \\
\hline \multicolumn{7}{|l|}{ TNM stage $(T)$} \\
\hline $1-2$ & 69 & $14(20)$ & $22(32)$ & $33(48)$ & \multirow{2}{*}{28.55} & \multirow{2}{*}{$\leq 0.001$} \\
\hline $3-4$ & 408 & $126(31)$ & $206(50)$ & $76(19)$ & & \\
\hline \multicolumn{7}{|l|}{ Lymph node metastasis } \\
\hline $\mathrm{N}_{0}$ & 290 & $66(23)$ & $140(48)$ & $84(29)$ & \multirow{2}{*}{23.088} & \multirow{2}{*}{$\leq 0.001$} \\
\hline $\mathrm{N}_{1-3}$ & 187 & $74(40)$ & $88(47)$ & $25(13)$ & & \\
\hline \multicolumn{7}{|c|}{ Location (miss samples, $N=286$ ) } \\
\hline Left & 49 & $17(35)$ & $23(47)$ & $9(18)$ & \multirow{2}{*}{1.123} & \multirow{2}{*}{0.570} \\
\hline Right & 142 & $38(27)$ & $74(52)$ & $30(21)$ & & \\
\hline
\end{tabular}

L: low expression; M: moderate expression; H: high expression; statistical method: chi-square test.

cells, our data showed that SDC1 was expressed at low levels in $29.4 \%$ (140/477) of CRCs, moderately expressed in $44.8 \%$ (228/477) of CRCs, and highly expressed in 22.8\% (109/477) of CRCs (Table 1). We next assessed the relationship between SDC1 expression and patients' clinicopathological characteristics. SDC1 expression was not correlated with age $(p=0.345)$, sex $(p=0.686)$, or tumor diameter $(p=0.232)$. However, loss of SDC1 protein was significantly associated with poor differentiation $(p=0.017)$, advanced TNM stage $(p \leq 0.001)$, and LN metastasis $(p \leq 0.001)$ (Table 2$)$.

3.3. Loss of SDC1 Expression Is Significantly Associated with Poor Prognosis of ESCC Patients. Kaplan-Meier analysis revealed that patients who exhibited reduced SDC1 (moderate or low) expression were associated with poorer overall survival and disease-free survival compared to patients who exhibited high SDC1 expression $(p=0.0045, p<0.0001$ and $p=0.0038, p<0.0001$, respectively, Figures 3(a) and 3(b)). These data indicate that SDC1 plays a role as a reliable tumor suppressor in CRC.

\section{Discussion}

Our findings derive from a large, clinically annotated tissue microarray of CRC specimens and add to the body of evidence that the loss of epithelial SDC1 is a general feature of carcinoma progression. The loss of SDC1 expression in local lymph node metastasis is evidence of the prometastasis function of SDC1. In fact, our results showed that the loss of expression of epithelial SDC1 truly correlates with poor dedifferentiation, stage, and local lymph node metastasis in CRC. In agreement with other analyses of CRC, the loss of epithelial SDC1 was correlated with tumor TNM 


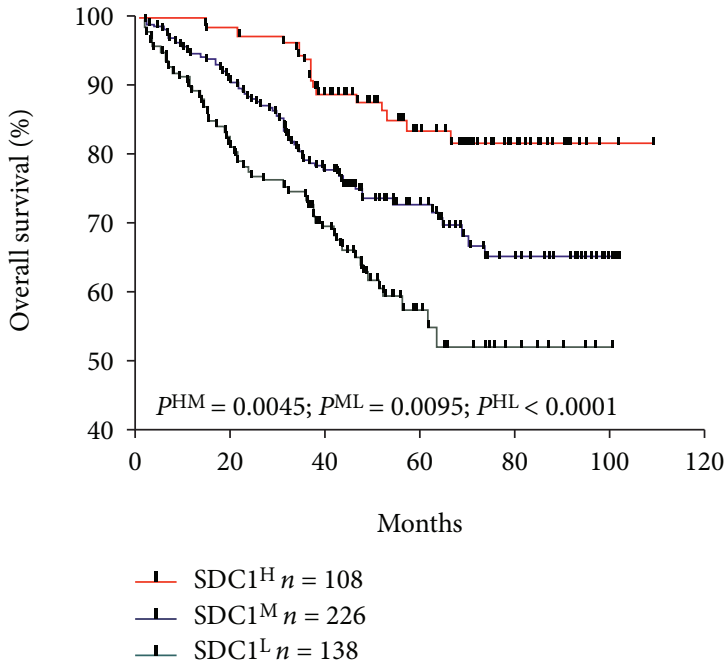

(a)

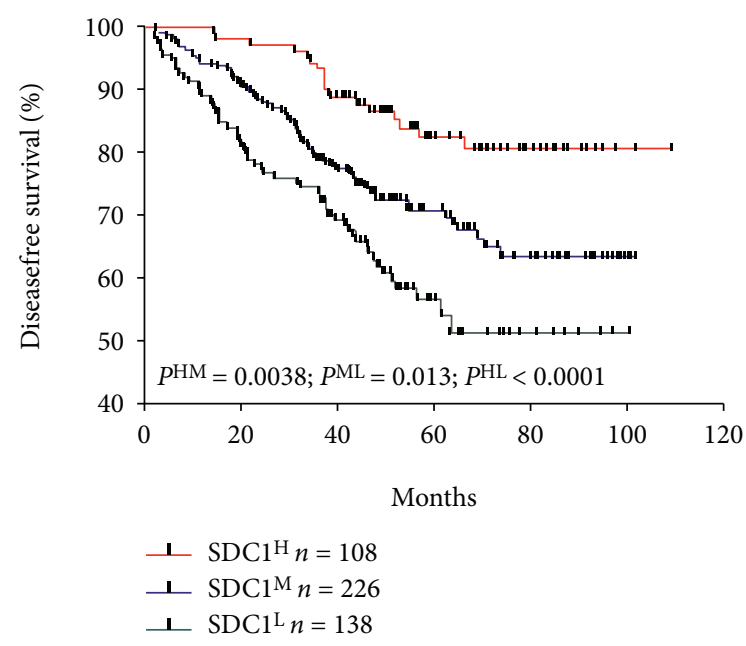

(b)

Figure 3: Relationship between CRC SDC1 status and patient survival. The Kaplan-Meier survival curves demonstrate that low, moderate, and high SDC1 expression in the tumors did correlate with (a) overall survival and (b) disease-free survival.

stage $[4-6,13]$, and the incidence of metastasis was correlated with local lymph nodes [4-6].

SDC3 and SDC4 have been reported as oncogenes $[15,16]$. Recently, syndecan-2 (SDC2) methylation was highlighted as a potential marker for early CRC detection. A DNA microarray analysis of neoplastic samples showed a high SDC2 methylation rate of approximately 95\%, regardless of the early colorectal cancer stage [17]. Blood SDC2 methylation data from 131 CRC patients and 125 healthy subjects showed a high sensitivity of $92.3 \%$ for detecting stage I CRCs [18]. Bowel lavage fluid (BLF) SDC2 methylation data showed that SDC2 methylation was positive in $100 \%$ of villous adenoma, high-grade dysplasia, and hyperplastic polyp samples; in $88.9 \%$ of tubular adenoma samples; and in $0 \%$ of normal mucosal samples [19]. These results suggest that the reduction in SDC1 expression in CRCs may also be caused by SDC1 DNA methylation, and further research is needed.

The syndecan transmembrane domain and transmembrane domain-induced dimerization seem to critically regulate various functions of syndecan family members [20]. Research has shown that SDC1 is coexpressed with EMT markers (E-cadherin and $\beta$-catenin) in CRCs and that this coexpression is regulated during epithelial-mesenchymal transition (EMT) [21]. The loss of SDC1 expression in carcinoma cells reduces cell adhesion to the extracellular matrix and enhances cell motility and invasion [22]. Our results showed that SDC1 expression was mainly in the form of a dimer in normal colorectal epithelial cells and was downregulated in CRCs. This suggests that SDC1 is inactivated in CRCs, thus reducing cell adhesion to the extracellular matrix and enhancing cell motility and invasion.

We also found that the loss of expression of epithelial SDC1 significantly correlates with poor patient survival. Previous studies reached conflicting conclusions on whether reduced SDC1 is correlated with decreased patient survival [4-6]. A study from Japan revealed that the low expression of epithelial SDC1 was significantly associated with poor clinical outcome in CRC [11], but two studies from Finland and the USA have shown that the low expression of epithelial SDC1 did not significantly correlate with the survival of CRC $[23,24]$. It is important to note that the studies that have examined the use of SDC1 as a prognostic marker were performed in different countries and on different continents [4-6]. Therefore, other factors such as treatment plans, genetic variations, and ethnicity may have influenced the results and affected the prognostic value of SDC1 in CRC progression and metastasis. Our data are in agreement with data from the study of Fujiya et al. [14]. The data suggest that the relationship between SDC1 expression and the prognosis of CRC patients may have ethnic and regional differences: the loss of SDC1 expression was correlated with a poor prognosis for East Asian CRC patients but not for Europeans.

In summary, the loss of SDC1 expression in CRC is closely associated with poor differentiation, stage, and local lymph node metastasis. SDC1 is a valuable biomarker for predicting the prognosis of CRC patients in northern China.

\section{Data Availability}

The data used to support the findings of this study are available from the corresponding author upon request.

\section{Conflicts of Interest}

The authors declare no conflicts of interest.

\section{Acknowledgments}

This research was supported by the National Natural Science Foundation of China (81802945), the Natural Science Foundation of Shandong Province, China (ZR2016HQ28); Science and Technology Development Project of Jining (2016-56-28), the PhD Research Foundation Affiliated 
Hospital of Jining Medical University (2016-BS-002); the cultivation project of the National Natural Science Foundation of Jining Medical University (JYP201731); research support fund for young teachers of Jining Medical University (JY2017FS007, JY2017FS001, JYFC2018FKJ036); Nursery Project of Affiliated Hospital of Jining Medical University (MP-2016-017; MP-2016-020).

\section{References}

[1] R. Siegel, C. Desantis, and A. Jemal, "Colorectal cancer statistics, 2014," CA: a Cancer Journal for Clinicians, vol. 64, no. 2, pp. 104-117, 2014.

[2] M. M. Center, A. Jemal, R. A. Smith, and E. Ward, "Worldwide variations in colorectal cancer," CA: a Cancer Journal for Clinicians, vol. 59, no. 6, pp. 366-378, 2009.

[3] E. Tkachenko, J. M. Rhodes, and M. Simons, "Syndecans: new kids on the signaling block," Circulation Research, vol. 96, no. 5, pp. 488-500, 2005.

[4] M. Bernfield, R. Kokenyesi, M. Kato et al., "Biology of the syndecans: a family of transmembrane heparan sulfate proteoglycans," Annual Review of Cell Biology, vol. 8, no. 1, pp. 365-393, 1992.

[5] A. N. Alexopoulou, H. A. Multhaupt, and J. R. Couchman, "Syndecans in wound healing, inflammation and vascular biology," The International Journal of Biochemistry \& Cell Biology, vol. 39, no. 3, pp. 505-528, 2007.

[6] R. Chakravarti and J. C. Adams, "Comparative genomics of the syndecans defines an ancestral genomic context associated with matrilins in vertebrates," BMC Genomics, vol. 7, no. 1, p. 83, 2006.

[7] M. D. Bass, M. R. Morgan, and M. J. Humphries, "Syndecans shed their reputation as inert molecules," Science Signaling, vol. 2, no. 64, p. pe18, 2009.

[8] A. Purushothaman, T. Uyama, F. Kobayashi et al., "Heparanase-enhanced shedding of syndecan-1 by myeloma cells promotes endothelial invasion and angiogenesis," Blood, vol. 115, no. 12, pp. 2449-2457, 2010.

[9] K. Hayashida, A. H. Bartlett, Y. Chen, and P. W. Park, "Molecular and cellular mechanisms of ectodomain shedding," The Anatomical Record: Advances in Integrative Anatomy and Evolutionary Biology, vol. 293, no. 6, pp. 925-937, 2010.

[10] X. Wang, D. Zuo, Y. Chen et al., "Shed Syndecan-1 is involved in chemotherapy resistance via the EGFR pathway in colorectal cancer," British Journal of Cancer, vol. 111, no. 10, pp. 1965-1976, 2014.

[11] O. P. Kallioniemi, U. Wagner, J. Kononen, and G. Sauter, "Tissue microarray technology for high-throughput molecular profiling of cancer," Human Molecular Genetics, vol. 10, no. 7, pp. 657-662, 2001.

[12] W. Wang, X. Li, W. Zhang et al., "Oxidored-nitro domain containing protein 1 (NOR1) expression suppresses slug/vimentin but not snail in nasopharyngeal carcinoma: inhibition of EMT in vitro and in vivo in mice," Cancer Letters, vol. 348, no. 1-2, pp. 109-118, 2014.

[13] R. M. Day, X. Hao, M. Ilyas, P. Daszak, I. C. Talbot, and A. Forbes, "Changes in the expression of syndecan-1 in the colorectal adenoma-carcinoma sequence," Virchows Archiv, vol. 434, no. 2, pp. 121-125, 1999.

[14] M. Fujiya, J. Watari, T. Ashida et al., "Reduced expression of syndecan-1 affects metastatic potential and clinical outcome in patients with colorectal cancer," Japanese Journal of Cancer Research, vol. 92, no. 10, pp. 1074-1081, 2001.

[15] Y. Yamada, T. Arai, S. Kojima et al., "Regulation of antitumor miR-144-5p targets oncogenes: direct regulation of syndecan-3 and its clinical significance," Cancer Science, vol. 109, no. 9, pp. 2919-2936, 2018.

[16] R. Leblanc, D. Sahay, A. Houssin, I. Machuca-Gayet, and O. Peyruchaud, "Autotaxin- $\beta$ interaction with the cell surface via syndecan- 4 impacts on cancer cell proliferation and metastasis," Oncotarget, vol. 9, no. 69, pp. 33170-33185, 2018.

[17] T. Oh, N. Kim, Y. Moon et al., "Genome-wide identification and validation of a novel methylation biomarker, SDC2, for blood-based detection of colorectal cancer," The Journal of Molecular Diagnostics, vol. 15, no. 4, pp. 498-507, 2013.

[18] S. M. Mitchell, T. Ho, G. S. Brown et al., "Evaluation of methylation biomarkers for detection of circulating tumor DNA and application to colorectal cancer," Genes, vol. 7, no. 12, 2016.

[19] Y. S. Park, D. S. Kim, S. W. Cho et al., "Analysis of syndecan-2 methylation in bowel lavage fluid for the detection of colorectal neoplasm," Gut and Liver, vol. 12, no. 5, pp. 508-515, 2018.

[20] B. Jang, H. Jung, H. Hong, and E. S. Oh, "Syndecan transmembrane domain modulates intracellular signaling by regulating the oligomeric status of the cytoplasmic domain," Cellular Signalling, vol. 52, pp. 121-126, 2018.

[21] A. Mitselou, V. Galani, U. Skoufi, D. L. Arvanitis, E. Lampri, and E. Ioachim, "Syndecan-1, epithelial-mesenchymal transition markers (E-cadherin/ $\beta$-catenin) and neoangiogenesisrelated proteins (PCAM-1 and endoglin) in colorectal cancer," Anticancer Research, vol. 36, no. 5, pp. 2271-2280, 2016.

[22] Y. H. Teng, R. S. Aquino, and P. W. Park, "Molecular functions of syndecan-1 in disease," Matrix Biology, vol. 31, no. 1, pp. 316, 2012.

[23] M. Lundin, S. Nordling, J. Lundin, J. Isola, J. P. Wiksten, and C. Haglund, "Epithelial syndecan-1 expression is associated with stage and grade in colorectal cancer," Oncology, vol. 68, no. 4-6, pp. 306-313, 2005.

[24] Y. Hashimoto, M. Skacel, and J. C. Adams, "Association of loss of epithelial syndecan-1 with stage and local metastasis of colorectal adenocarcinomas: an immunohistochemical study of clinically annotated tumors," BMC Cancer, vol. 8, no. 1, 2008 . 


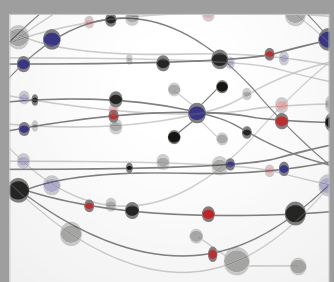

The Scientific World Journal
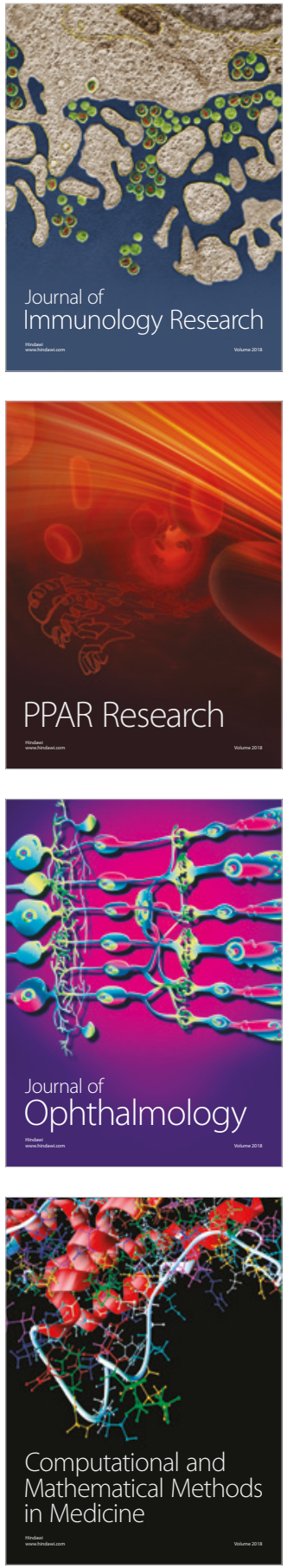

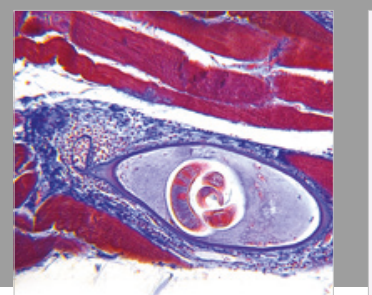

Gastroenterology Research and Practice

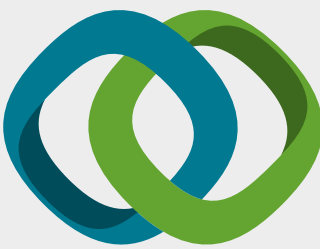

\section{Hindawi}

Submit your manuscripts at

www.hindawi.com
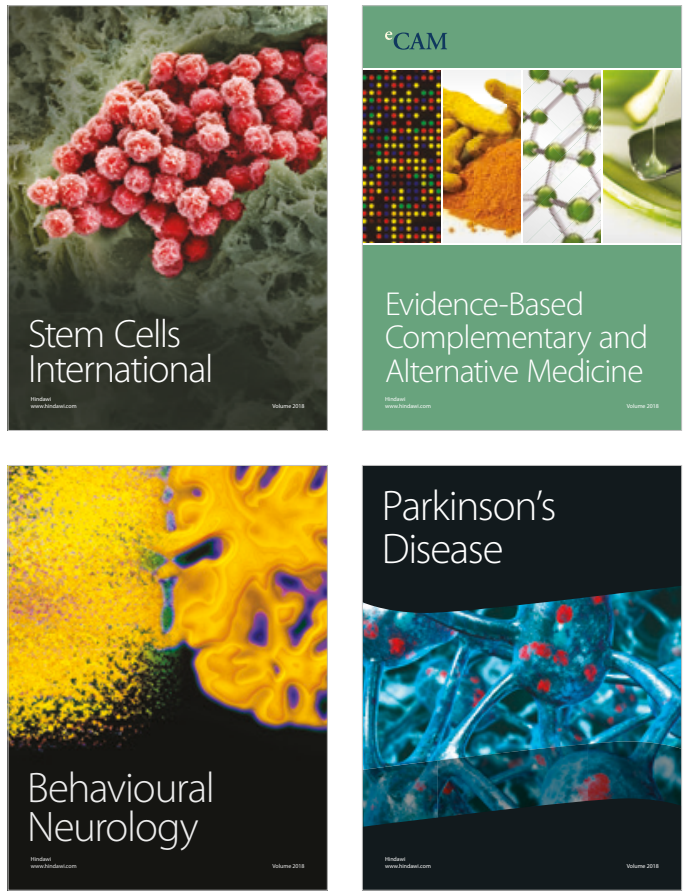

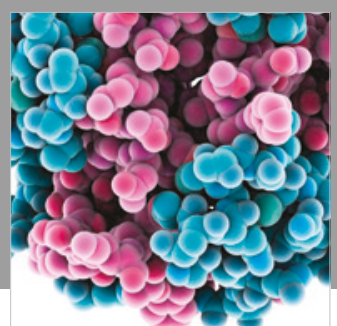

ournal of

Diabetes Research

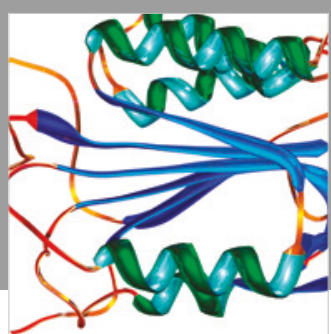

Disease Markers
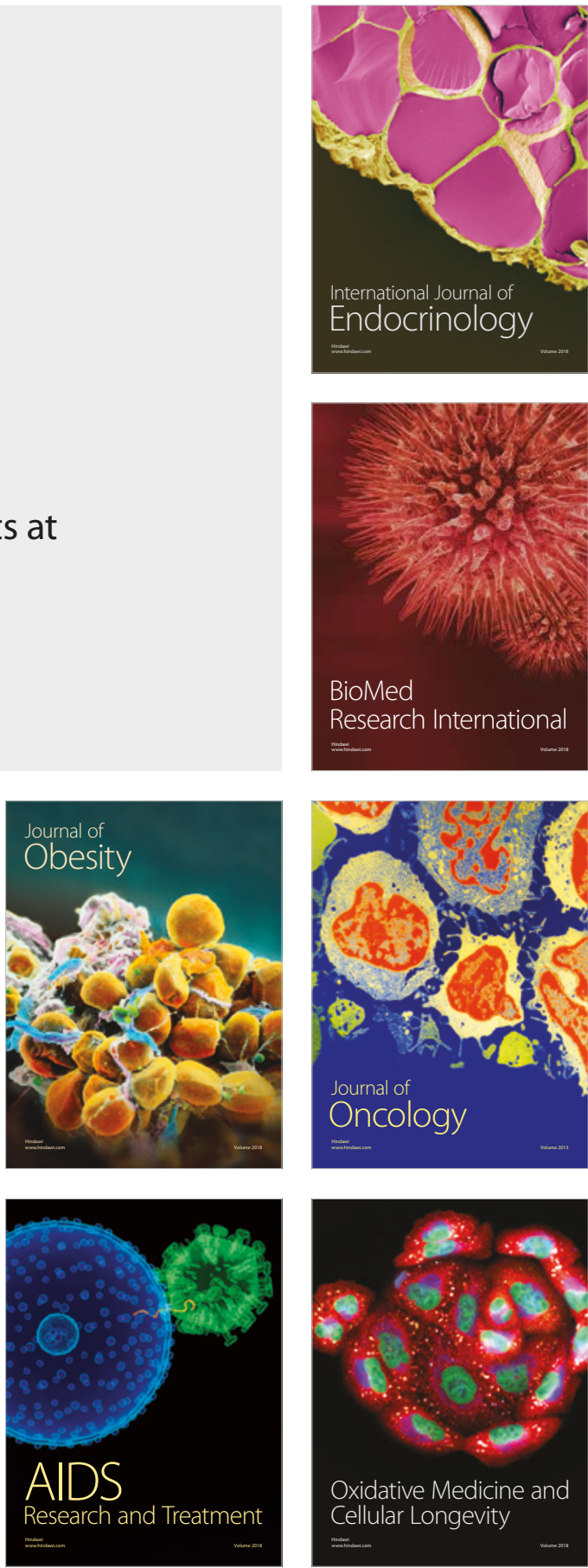